\title{
EGU2020-20261
}

https://doi.org/10.5194/egusphere-egu2020-20261

EGU General Assembly 2020

(c) Author(s) 2020. This work is distributed under

the Creative Commons Attribution 4.0 License.

\section{Root traits as key proxies to unravel plant and ecosystem functioning: entities, trait selection and outlook}

Boris Rewald ${ }^{1}$, Grégoire T. Freschet ${ }^{2,3}$, Catherine Roumet ${ }^{2}$, Alexia Stokes ${ }^{2}$, Monique Weemstra², Richard D. Bardgett ${ }^{4}$, A. Glyn Bengough ${ }^{5,6}$, Louise H. Comas ${ }^{7}$, Gerlinde B. De Deyn ${ }^{8}$, David Johnson ${ }^{4}$, Jitka Klimešová ${ }^{9}$, Martin Lukac ${ }^{10}, M$. Luke McCormack ${ }^{11}$, Ina C. Meier ${ }^{12}$, Loïc Pagès ${ }^{13}$, Hendrik Poorter $^{14,15}$, Ivan Prieto ${ }^{16}$, Nina Wurzburger ${ }^{17}$, and Marcin Zadworny ${ }^{18}$

${ }^{1}$ Forest and Soil Sciences, University of Natural Resources and Life Sciences, Vienna, Austria (boris.rewald@boku.ac.at) ${ }^{2}$ Centre d'Ecologie Fonctionnelle et Evolutive, UMR 5175 (CNRS - Université de Montpellier - Université Paul-Valéry Montpellier - EPHE - IRD), Montpellier, France

${ }^{3}$ Station d'Ecologie Théorique et Expérimentale, CNRS, Moulis, France

${ }^{4}$ School of Earth and Environmental Sciences, University of Manchester, Manchester, UK

5James Hutton Institute, Invergowrie Dundee, UK

${ }^{6}$ School of Engineering, Mathematics and Physics, University of Dundee, Dundee, UK

${ }^{7}$ Water Management Research Unit, USDA-ARS, Fort Collins, USA

${ }^{8}$ Soil Biology and Biological Soil Quality Group, Wageningen University, Wageningen, The Netherlands

${ }^{9}$ Institute of Botany, Czech Academy of Sciences, Třeboň, Czech Republic

${ }^{10}$ School of Agriculture, Policy \& Development, University of Reading, Reading, UK

${ }^{11}$ Center for Tree Science, Morton Arboretum, Lisle, USA

${ }^{12}$ Plant Ecology, University of Goettingen, Göttingen, Germany

${ }^{13}$ Plantes et Systèmes de culture Horticoles, INRA UR 1115, Avignon, France

${ }^{14}$ Plant Sciences (IBG-2), Forschungszentrum Jülich GmbH, Jülich, Germany

${ }^{15}$ Department of Biological Sciences, Macquarie University, North Ryde, Australia

${ }^{16}$ Centro de Edafología y Biología Aplicada del Segura (CSIC), Campus Universitario de Espinardo, Murcia, Spain

${ }^{17}$ Odum School of Ecology, University of Georgia, Athens, USA

${ }^{18}$ Institute of Dendrology, Polish Academy of Sciences, Kórnik, Poland

Root systems show a tremendous diversity both between and within species, suggesting a large variability in plant functioning and effects on ecosystem properties and processes. In recent decades, developments in many areas of root research have brought considerable advances in our understanding of root traits and their contribution to plant and ecosystem functioning. However, despite major progress, a comprehensive overview-bridging research fields-is lacking. Furthermore, considerable uncertainties exist in the identification of root entities, and the selection and standardized measurement of traits. Here, we provide a comprehensive overview on root entities, exemplify recent advances in our understanding of both theoretical and demonstrated relationships between root traits and plant or ecosystem functioning, discuss traittrait relationships and hierarchies among traits, and critically assess current strengths and gaps in our knowledge. 
